Journal of the Operations Research

Society of Japan

Vol. 41, No. 1, March 1998

\title{
A COMPOSITE QUEUE WITH VACATION/SET-UP/CLOSE-DOWN TIMES FOR SVCC IN IP OVER ATM NETWORKS
}

\author{
Yutaka Sakai \\ Kyoto University
}

\author{
Yoshitaka Takahashi \\ NTT Multimedia \\ Networks Laboratories
}

\author{
Yutaka Takahashi \\ Nara Institute of \\ Science and Technolgoy
}

\author{
Toshiharu Hasegawa \\ Kyoto University
}

(Received May 2, 1997; Revised September 17, 1997)

\begin{abstract}
We consider a finite-capacity $\mathrm{M} / \mathrm{G} / 1 / \mathrm{K}$ queueing system with a mixture of the single vacation, close-down, and set-up times; arising out of the switched virtual channel connection (SVCC) mechanism for an IP over ATM network. Applying the supplementary variable approach, we derive the steady-state queue length and waiting time distributions, as well as the set-up occurring rate. Taking the limit of our results as the capacity $(\mathrm{K})$ tends to infinity, we obtain the results for an infinite-capacity queueing system, covering the previous work by Hassan and Atiquzzaman as a special case.
\end{abstract}

\section{Introduction}

Teletraffic engineers frequently encounter queueing situations with vacation time and exhaustive service discipline. By vacation time, we mean that the server becomes unavailable for occasional intervals of time, and by exhaustive we mean that customers are served continuously until there is no customer in the system. A classical example for this situation is the time division multiple access (TDMA) scheme where the vacation time corresponds to a constant slotted time period in the TDMA system.

Under the exhaustive service discipline, the well known stochastic decomposition formula will be useful for infinite capacity queues; see Doshi [1] and Miyazawa [5]. However, if we would like to evaluate the loss probability we have to treat a finite capacity system rather than infinite capacity systems.

Assuming a Poisson input and a finite capacity queue, Lee [4] provided a numerical algorithm for this system via the embedded Markov chain technique; see also Frey and Takahashi [2] for a more simplified analysis for the $\mathrm{M} / \mathrm{G} / 1 / \mathrm{K}$ queue with vacation time and exhaustive service discipline.

Very recently in the IP over ATM networks, we can see a more complicated queueing situation where the close-down time as well as the set-up time are further needed. The close-down time here corresponds to an inactivity timer in the switched virtual channel connections (SVCC) environment (see Hassan and Atiquzzaman [3]). Also, the set-up time cannot be negligible for the ATM server. An approximate queueing result (using the M/G/1 queue) has been reported in Hassan and Atiquzzaman [3]. Their approximation is based on i.i.d. service time of each packet to obtain the first and second moments of the waiting time. However, under the existance of SET-UP time, this i.i.d. service time does not hold.

The main goal of this paper is 1) to present a new finite capacity queueing system with vacation, set-up, and close-down times for the SVCC operation in IP over ATM networks, generalizing the queueing system of Hassan and Atiquzzaman; 2) and to provide an exact result for the generalized system. The approach taken here is the supplementary variable 
technique. It should be noted that our approach enables one to treat the infinite capacity system by taking the limit of our result as the capacity $(K)$ tends to infinity. We can derive an exact formula to the Hassan and Atiquzzaman's system [3] for a special case (the infinite queue without vacation).

This paper is organized as follows. We describe the model and introduce notations in Section 2. Section 3 derives a set of Laplace-Stieltjes transform equations through the supplementary variable approach. Section 4 is then devoted to the numerical algorithm procedure for the steady-state probabilities. The procedure is expressed step by step to show our algorithm. Using these results, we express performance measures such as the mean waiting time, set-up rate, and loss probability in Section 5 and Section 6 presents the numerical results for the performance measures. Section 7 shows that the limiting behavior of the finite capacity system is consistent with that of the infinite capacity system analyzed by the imbedded Markov chain approach.

\section{The model}

We consider an $M / G / 1 / K$ system with single vacation, set-up and close-down time, where $\mathrm{K}$ represents the maximum number of customers allowed in the system. The system consists of the waiting room and the server. We assume a customer arrives at the system according to a Poisson process with intensity $\lambda$ independent of the system state. These arriving customers are served under the First-In-First-Out (FIFO) discipline, where the customers will be served in order of their arrivals to the system. Since we consider the system with a finite capacity, a customer will be lost if it finds the waiting room all occupied. We assume the exhaustive service discipline and the server continues to serve the customers in the queue until all of them are served. The service times form a sequence of i.i.d. random variables with distribution function (DF) $B(x)$ and the Laplace-Stieltjes transform (LST) $B^{*}(s) \equiv \int_{0}^{\infty} e^{-s x} B(d x)$.

We call the period where the server is working a busy period. If the server finds that there is no customer in the queue at a service completion, the system goes into a close-down period with DF $C(x)$ and LST $C^{*}(s)$. During the close-down period, if a customer arrives, the server immediately begins service for that customer without set-up period and another busy period begins. On the other hand, if no customer arrives until the end of a close-down period, the system goes into a single vacation period. At the end of a vacation period, if the server finds one or more customers waiting, the server begins to serve for them after a set-up period. We denote by $S(x), S^{*}(s)\left(V(x), V^{*}(s)\right)$ the DF or the LST of the set-up period length (vacation period length). If the server finds no customers waiting, the system goes into an idle period and an arrival of a customer ends this period. Then the server begins to serve after a set-up period. It should be noted that our model reduces to that of Hassan and Atiquzzaman [3] if the capacity $(\mathrm{K})$ is infinite and the vacation length is zero. We define $\hat{B}$ as the remaining service time. Similarly we define $\hat{V}, \hat{S}$ and $\hat{C}$.

We further need the following notations for our subsequent analysis.

$L$ : number of customers in the system including the one in the server.

$$
\begin{aligned}
\Pi_{B, i}(x) d x & \equiv \operatorname{Prob}[L=i, x<\hat{B} \leq x+d x, \text { the system is busy }] \\
\Pi_{V, i}(x) d x & \equiv \operatorname{Prob}[L=i, x<\hat{V} \leq x+d x, \text { the system is in vacation] } \\
\Pi_{S, i}(x) d x & \equiv \operatorname{Prob}[L=i, x<\hat{S} \leq x+d x, \text { the system is setting-up] }
\end{aligned}
$$




$$
\begin{array}{rlrl}
\Pi_{C, i}(x) d x & \equiv \operatorname{Prob}[L=i, x<\hat{C} \leq x+d x, \text { the system is closing-down }] \\
\Pi_{B, i}^{*}(s) & \equiv \int_{0}^{\infty} e^{-s x} \Pi_{B, i}(d x) & \Pi_{V, i}^{*}(s) \equiv \int_{0}^{\infty} e^{-s x} \Pi_{V, i}(d x) \\
\Pi_{S, i}^{*}(s) & \equiv \int_{0}^{\infty} e^{-s x} \Pi_{S, i}(d x) & \Pi_{C, i}^{*}(s) \equiv \int_{0}^{\infty} e^{-s x} \Pi_{C, i}(d x)
\end{array}
$$

\section{Supplementary variable approach}

In this section, we obtain Laplace-Stieltjes transforms, $\Pi_{C, 0}^{*}(s), \Pi_{V, i}^{*}(s)(0 \leq i \leq K)$, $\Pi_{S, i}^{*}(s)(1 \leq i \leq K)$, and $\Pi_{B, i}^{*}(s)(1 \leq i \leq K)$, which are the bases of analysis in the following section.

Since the service, vacation, set-up, close-down times are generally distributed (having non-Markovian property), the queue length process does not form any Markov chain. Here, the supplementary variable technique is applied to make our system Markovian. To be more exact, the joint distribution of the queue length and the remaining service (vacation, set-up, or close-down) time forms a Markov process. Observing the system state at time $t$ and $t+\Delta t$, we have following equations.

$$
\begin{aligned}
& \frac{d \Pi_{C, 0}(x)}{d x}=\lambda \Pi_{C, 0}(x)-\Pi_{B, 1}(0) \frac{C(d x)}{d x} \\
& \frac{d \Pi_{V, 0}(x)}{d x}=\lambda \Pi_{V, 0}(x)-\Pi_{C, 0}(0) \frac{V(d x)}{d x} \\
& \frac{d \Pi_{V, i}(x)}{d x}=\lambda \Pi_{V, i}(x)-\lambda \Pi_{V, i-1}(x), \quad \text { for } i=1, \cdots, K-1 \\
& \frac{d \Pi_{V, K}(x)}{d x}=-\lambda \Pi_{V, K-1}(x) \\
& \frac{d \Pi_{S, 1}(x)}{d x}=\lambda \Pi_{S, 1}(x)-\left(\lambda \Pi_{0}^{*}+\Pi_{V, 1}(0)\right) \frac{S(d x)}{d x} \\
& \frac{d \Pi_{S, i}(x)}{d x}=\lambda \Pi_{S, i}(x)-\lambda \Pi_{S, i-1}(x)-\Pi_{V, i}(0) \frac{S(d x)}{d x}, \quad \text { for } i=2, \cdots, K-1 \\
& \frac{d \Pi_{S, K}(x)}{d x}=-\lambda \Pi_{S, K-1}(x) \\
& \frac{d \Pi_{B, 1}(x)}{d x}=\lambda \Pi_{B, 1}(x)-\left(\Pi_{B, 2}(0)+\Pi_{S, 1}(0)+\Pi_{C, 1}(0)\right) \frac{B(d x)}{d x} \\
& \frac{d \Pi_{B, i}(x)}{d x}=\lambda \Pi_{B, i}(x)-\lambda \Pi_{B, i-1}(x)-\left(\Pi_{B, i+1}(0)+\Pi_{S, i}(0)\right) \frac{B(d x)}{d x}, \quad \text { for } i=2, \cdots, K-1 \\
& \frac{d \Pi_{B, K}(x)}{d x}=-\lambda \Pi_{B, K-1}(x)-\Pi_{S, K}(0) \frac{B(d x)}{d x}
\end{aligned}
$$

where $\Pi_{0}^{*}$ is the probability that the system is idle at an arbitrary time. Equating the rate at which a vacation period ends with no customers present to the rate at which the idle period following ends because of arrivals, we have

$$
\lambda \Pi_{0}^{*}=\Pi_{V, 0}(0) .
$$

During the close-down time, an arriving customer may end the period, thus

$$
\Pi_{C, 1}(\theta)=\lambda \int_{0}^{\infty} \Pi_{C, 0}(x) d x
$$


Taking into account (1) and (2), we obtain Laplace-Stieltjes transforms,

$$
\begin{aligned}
(\lambda-s) \Pi_{C, 0}^{*}(s) & =-\Pi_{C, 0}(0)+\Pi_{B, 1}(0) C^{*}(s) \\
(\lambda-s) \Pi_{V, 0}^{*}(s) & =-\Pi_{V, 0}(0)+\Pi_{C, 0}(0) V^{*}(s) \\
(\lambda-s) \Pi_{V, i}^{*}(s) & =-\Pi_{V, i}(0)+\lambda \Pi_{V, i-1}^{*}(s), \quad \text { for } 1 \leq i \leq K-1 \\
-s \Pi_{V, K}^{*}(s) & =-\Pi_{V, K}(0)+\lambda \Pi_{V, K-1}^{*}(s) \\
(\lambda-s) \Pi_{S, 1}^{*}(s) & =-\Pi_{S, 1}(0)+\left(\Pi_{V, 0}(0)+\Pi_{V, 1}(0)\right) S^{*}(s) \\
(\lambda-s) \Pi_{S, i}^{*}(s) & =-\Pi_{S, i}(0)+\lambda \Pi_{S, i-1}^{*}(s)+\Pi_{V, i}(0) S^{*}(s), \text { for } 2 \leq i \leq K-1 \\
-s \Pi_{S, K}^{*}(s) & =-\Pi_{S, K}(0)+\lambda \Pi_{S, K-1}^{*}(s)+\Pi_{V, K}(0) S^{*}(s) \\
(\lambda-s) \Pi_{B, 1}^{*}(s) & =-\Pi_{B, 1}(0)+\left(\Pi_{B, 2}(0)+\Pi_{S, 1}(0)+\lambda \Pi_{C, 0}^{*}(0)\right) B^{*}(s) \\
(\lambda-s) \Pi_{B, i}^{*}(s) & =-\Pi_{B, i}(0)+\lambda \Pi_{B, i-1}^{*}(s)+\left(\Pi_{B, i+1}(0)+\Pi_{S, i}(0)\right) B^{*}(s), \\
-s \Pi_{B, K}^{*}(s) & =-\Pi_{B, K}(0)+\lambda \Pi_{B, K-1}^{*}(s)+\Pi_{S, K}(0) B^{*}(s) .
\end{aligned}
$$

\section{System state probability}

In this section we give $\Pi_{C, 0}(0), \Pi_{C, 0}^{*}(0), \Pi_{V, i}(0)(0 \leq i \leq K), \Pi_{V, i}^{*}(0)(0 \leq i \leq K)$, $\Pi_{S, i}(0)(1 \leq i \leq K), \Pi_{S, i}^{*}(0)(1 \leq i \leq K), \Pi_{B, i}(0)(1 \leq i \leq K)$ and $\Pi_{B, i}^{*}(0)(1 \leq i \leq K)$. From these results we obtain the system state probability at which close-down/vacation/setup/service/idle periods end and at an arbitrary point. In the following we express all probabilities in terms of $\Pi_{B, 1}(0)$. At last we have $\Pi_{B, 1}(0)$ from the normalization condition. $4.1 \Pi_{C, 0}(0)$ and $\Pi_{C, 0}^{*}(0)$ in terms of $\Pi_{B, 1}(0)$

We express $\Pi_{C, 0}(0)$ and $\Pi_{C, 0}^{*}(0)$ in terms of $\Pi_{B, 1}(0)$. Substituting $s=\lambda$ and $s=0$ into (3) yields

$$
\begin{aligned}
\Pi_{C, 0}(0) & =\Pi_{B, 1}(0) C^{*}(\lambda) \\
\lambda \Pi_{C, 0}^{*}(0) & =-\Pi_{C, 0}(0)+\Pi_{B, 1}(0) \\
& =\left(1-C^{*}(\lambda)\right) \Pi_{B, 1}(0) .
\end{aligned}
$$

$4.2 \Pi_{V, i}(0)(1 \leq i \leq K)$ and $\Pi_{V, i}^{*}(0)(1 \leq i \leq K)$ in terms of $\Pi_{B, 1}(0)$

We then express $\Pi_{V, i}(0)(1 \leq i \leq K)$ and $\Pi_{V, i}^{*}(0)(1 \leq i \leq K)$ in terms of $\Pi_{B, 1}(0)$. Substituting $s=\lambda$ into (4) and (5), we have

$$
\begin{aligned}
\Pi_{V, 0}(0) & =\Pi_{B, 1}(0) C^{*}(\lambda) V^{*}(\lambda) \\
\Pi_{V, i}(0) & =\lambda \Pi_{V, i-1}^{*}(\lambda), \quad \text { for } 1 \leq i \leq K-1 .
\end{aligned}
$$

Differentiating (4) and (5), and inserting $s=\lambda$, we have

$$
\begin{aligned}
& -(n+1) \Pi_{V, 0}^{*(n)}(\lambda)=\Pi_{B, 1}(0) C^{*}(\lambda) V^{*(n+1)}(\lambda), \quad \text { for } n \geq 0 \\
& -(n+1) \Pi_{V, i}^{*(n)}(\lambda)=\lambda \Pi_{V, i-1}^{*(n+1)}(\lambda), \quad \text { for } n \geq 0, i \geq 1 .
\end{aligned}
$$

Using (17) and (18), we have

$$
\Pi_{V, i}^{*}(\lambda)=(-1)^{i+1} \frac{\lambda^{i}}{(i+1) !} \Pi_{B, 1}(0) C^{*}(\lambda) V^{*(i+1)}(\lambda), \quad \text { for } 0 \leq i \leq K-2 .
$$


From $(19),(15)$ and $(16)$ we can express $\Pi_{V, i}(0)(0 \leq i \leq K-1)$ in terms of $\Pi_{B, 1}(0)$. Indeed we have

$$
\begin{aligned}
& \Pi_{V, 0}(0)=\Pi_{B, 1}(0) C^{*}(\lambda) V^{*}(\lambda) \\
& \Pi_{V, i}(0)=(-1)^{i} \frac{\lambda^{i}}{(i) !} \Pi_{B, 1}(0) C^{*}(\lambda) V^{*(i)}(\lambda), \quad \text { for } 1 \leq i \leq K-1 .
\end{aligned}
$$

Next we substitute $s=0$ into (4), (5) and (6), yielding

$$
\begin{aligned}
\lambda \Pi_{V, 0}^{*}(0) & =-\Pi_{V, 0}(0)+\Pi_{B, 1}(0) C^{*}(\lambda) \\
\lambda \Pi_{V, i}^{*}(0) & =-\Pi_{V, i}(0)+\lambda \Pi_{V, i-1}^{*}(0), \quad \text { for } 1 \leq i \leq K-1 \\
\Pi_{V, K}(0) & =\lambda \Pi_{V, K-1}^{*}(0) .
\end{aligned}
$$

Since we have $\Pi_{V, i}(0)(1 \leq i \leq K-1)$ in terms of $\Pi_{B, 1}(0)$ we can easily express $\Pi_{V, i}^{*}(0)(0 \leq$ $i \leq K-1)$ in terms of $\Pi_{B, 1}(0)$ using (22) and (23). Together with these results and (24), we have $\Pi_{V, K}(0)$ in terms of $\Pi_{B, 1}(0)$

$$
\Pi_{V, K}(0)=-\sum_{i=0}^{K-1} \Pi_{V, i}(0)+\Pi_{B, 1}(0) C^{*}(\lambda) .
$$

Differentiating (4), (5) and (6), and substituting $s=0$ yield

$$
\begin{aligned}
\lambda \Pi_{V, 0}^{*(1)}(0) & =\Pi_{V, 0}^{*}(0)+\Pi_{B, 1}(0) C^{*}(\lambda) V^{*(1)}(0) \\
\lambda \Pi_{V, i}^{*(1)}(0) & =\Pi_{V, i}^{*}(0)+\lambda \Pi_{V, i-1}^{*(1)}(0), \quad \text { for } 1 \leq i \leq K-1 \\
-\Pi_{V, K}^{*}(0) & =\lambda \Pi_{V, K-1}^{*(1)}(0) .
\end{aligned}
$$

From these results, we can express $\Pi_{V, K}^{*}(0)$ in terms of $\Pi_{B, 1}(0)$

$$
\Pi_{V, K}^{*}(0)=-\left(\sum_{i=0}^{K-1} \Pi_{V, i}^{*}(0)+\Pi_{B, 1}(0) C^{*}(\lambda) V^{*(1)}(0)\right) .
$$

Hereby we complete our procedure.

$4.3 \Pi_{S, i}(0)(1 \leq i \leq K)$ and $\Pi_{S, i}^{*}(0)(1 \leq i \leq K)$ in terms of $\Pi_{B, 1}(0)$

We are next in a position to express $\Pi_{S, i}(0)(1 \leq i \leq K)$ and $\Pi_{S, i}^{*}(0)(1 \leq i \leq K)$ in terms of $\Pi_{B, 1}(0)$. Substituting $s=\lambda$ into $(7)$ and (8) yields

$$
\begin{aligned}
& \Pi_{S, 1}(0)=\left(\Pi_{V, 0}(0)+\Pi_{V, 1}(0)\right) S^{*}(\lambda) \\
& \Pi_{S, i}(0)=\lambda \Pi_{S, i-1}^{*}(\lambda)+\Pi_{V, i}(0) S^{*}(\lambda), \text { for } 2 \leq i \leq K-1 .
\end{aligned}
$$

Differentiating (7) and (8), and substituting $s=\lambda$, we have

$$
\begin{aligned}
& -(n+1) \Pi_{S, 1}^{*(n)}(\lambda)=\left(\Pi_{V, 0}(0)+\Pi_{V, 1}(0)\right) S^{*(n+1)}(\lambda), \quad \text { for } n \geq 0 \\
& -(n+1) \Pi_{S, i}^{*(n)}(\lambda)=\lambda \Pi_{S, i-1}^{*(n+1)}(\lambda)+\Pi_{V, i}(0) S^{*(n+1)}(\lambda), \quad \text { for } n \geq 0, i \geq 1 .
\end{aligned}
$$

Using (32) and (33), we have

$$
\begin{gathered}
\Pi_{S, i}^{*}(\lambda)=(-1)^{i} \frac{\lambda^{i-1}}{(i) !}\left(\Pi_{V, 0}(0)+\Pi_{V, 1}(0)\right) S^{*(i)}(\lambda)+\sum_{j=0}^{i-2}(-1)^{j+1} \frac{\lambda^{j}}{(j+1) !} \Pi_{V, i-j}(0) S^{*(j+1)}(\lambda) \\
\text { for } 2 \leq i \leq K-2
\end{gathered}
$$


From $(34),(30)$ and $(31)$, we can express $\Pi_{S, i}(0)(1 \leq i \leq K-1)$ in terms of $\Pi_{B, 1}(0)$ and $\Pi_{V, i}(0)(1 \leq i \leq K)$. We obtain

$$
\begin{aligned}
& \Pi_{S, 1}(0)=\left(\Pi_{V, 0}(0)+\Pi_{V, 1}(0)\right) S^{*}(\lambda) \\
& \Pi_{S, 2}(0)=-\lambda\left(\Pi_{V, 0}(0)+\Pi_{V, 1}(0)\right) S^{(1) *}(\lambda)+\Pi_{V, 2}(0) S^{*}(\lambda)
\end{aligned}
$$

and

$$
\begin{aligned}
& \Pi_{S, i}(0)=\lambda\left\{(-1)^{i-1} \frac{\lambda^{i-2}}{(i-1) !}\left(\Pi_{V, 0}(0)+\Pi_{V, 1}(0)\right) S^{*(i-1)}(\lambda)\right. \\
&+\left.\sum_{j=0}^{i-3}(-1)^{j+1} \frac{\lambda^{j}}{(j+1) !} \Pi_{V, i-1-j}(0) S^{*(j+1)}(\lambda)\right\}+\Pi_{V, i}(0) S^{*}(\lambda), \\
& \quad \text { for } 3 \leq i \leq K-1 .
\end{aligned}
$$

Remember that we have already expressed $\Pi_{V, i}(0)(1 \leq i \leq K)$ in terms of $\Pi_{B, 1}(0)$. Therefore, from (35), (36) and (37), we can express $\Pi_{S, i}(0)(1 \leq i \leq K-1)$ in terms of $\Pi_{B, 1}(0)$. As before, substituting $s=0$ into (7), (8) and (9) yields

$$
\begin{aligned}
\lambda \Pi_{S, 1}^{*}(0) & =-\Pi_{S, 1}(0)+\Pi_{V, 0}(0)+\Pi_{V, 1}(0) \\
\lambda \Pi_{S, i}^{*}(0) & =-\Pi_{S, i}(0)+\lambda \Pi_{S, i-1}^{*}(0)+\Pi_{V, i}(0), \quad \text { for } 2 \leq i \leq K-1 \\
\Pi_{S, K}(0) & =\lambda \Pi_{S, K-1}^{*}(0)+\Pi_{V, K}^{*}(0) .
\end{aligned}
$$

Since we have $\Pi_{S, i}(0)(1 \leq i \leq K-1)$ in terms of $\Pi_{B, 1}(0)$, we can easily express $\Pi_{S, i}^{*}(0)(0 \leq$ $i \leq K-1)$ in terms of $\Pi_{B, 1}(0)$ using (38) and (39). Together with these results and (40), we have $\Pi_{S, K}(0)$ in terms of $\Pi_{B, 1}(0)$.

$$
\Pi_{S, K}(0)=-\sum_{i=1}^{K-1} \Pi_{S, i}(0)+\sum_{i=0}^{K-1} \Pi_{V, i}(0)+\Pi_{V, K}^{*}(0) .
$$

At last, differentiating (7), (8) and (9) and substituting $s=0$ yield

$$
\begin{aligned}
\lambda \Pi_{S, 1}^{*(1)}(0) & =\Pi_{S, 1}^{*}(0)+\left(\Pi_{V, 0}(0)+\Pi_{V, 1}(0)\right) S^{*(1)}(0) \\
\lambda \Pi_{S, i}^{*(1)}(0) & =\Pi_{S, i}^{*}(0)+\lambda \Pi_{S, i-1}^{*(1)}(0)+\Pi_{V, i}(0) S^{*(1)}(0), \quad \text { for } 2 \leq i \leq K-1 \\
-\Pi_{S, K}^{*}(0) & =\lambda \Pi_{S, K-1}^{*(1)}(0)+\Pi_{V, K}(0) S^{*(1)}(0) .
\end{aligned}
$$

From these results, we can express $\Pi_{S, K}^{*}(0)$ using $\Pi_{B, 1}(0)$

$$
\Pi_{S, K}^{*}(0)=-\left(\sum_{i=1}^{K-1} \Pi_{S, i}^{*}(0)+\sum_{i=0}^{K} \Pi_{V, i}^{*}(0) S^{*(1)}(0)\right) .
$$

$4.4 \Pi_{B, i}(0)(1 \leq i \leq K)$ and $\Pi_{B, i}^{*}(0)(1 \leq i \leq K)$ in terms of $\Pi_{B, 1}(0)$

We now express $\Pi_{B, i}(0)(1 \leq i \leq K)$ and $\Pi_{B, i}^{*}(0)(1 \leq i \leq K)$ in terms of $\Pi_{B, 1}(0)$. Substituting $s=\lambda$ into (10) and (11), we have lastly

$$
\begin{aligned}
& \Pi_{B, 2}(0)=\frac{\Pi_{B, 1}(0)}{B^{*}(\lambda)}-\Pi_{S, 1}(0)-\Pi_{B, 1}(0)\left(1-C^{*}(\lambda)\right) \\
& \Pi_{B, i}(0)=\frac{\Pi_{B, i-1}(0)-\lambda \Pi_{B, i-2}^{*}(\lambda)}{B^{*}(\lambda)}-\Pi_{S, i-1}(0), \quad \text { for } 3 \leq i \leq K .
\end{aligned}
$$


Differentiating (10) and (11), then substituting $s=\lambda$, we have

$$
\begin{aligned}
& -(n+1) \Pi_{B, 1}^{*(n)}(\lambda)=\frac{\Pi_{B, 1}(0)}{B^{*}(\lambda)} B^{*(n+1)}(\lambda), \text { for } n \geq 0 \\
& -(n+1) \Pi_{B, i}^{*(n)}(\lambda)=\lambda \Pi_{B, i-1}^{*(n+1)}(\lambda)+\left(\Pi_{B, i+1}(0)+\Pi_{S, i}(0)\right) B^{*(n+1)}(\lambda), \text { for } n \geq 0, i \geq 1 .
\end{aligned}
$$

Using (48) and (49), we have

$$
\begin{aligned}
& \Pi_{B, i}^{*}(\lambda)=(-1)^{i} \frac{\lambda^{i-1}}{(i) !} \frac{\Pi_{B, 1}(0)}{B^{*}(\lambda)} B^{*(i)}(\lambda) \\
&+\sum_{j=0}^{i-2}(-1)^{j+1} \frac{\lambda^{j}}{(j+1) !}\left(\Pi_{B, i+1-j}(0)+\Pi_{S, i-j}(0)\right) B^{*(j+1)}(\lambda), \\
& \quad \text { for } 2 \leq i \leq K-2 .
\end{aligned}
$$

From $(50),(46)$ and $(47)$, we can express $\Pi_{B, i}(0)(2 \leq i \leq K-1)$ in terms of $\Pi_{B, 1}(0)$ and $\Pi_{S, i}(0)(1 \leq i \leq K)$. Thus we have

$$
\begin{aligned}
& \Pi_{B, 2}(0)=\frac{\Pi_{B, 1}(0)}{B^{*}(\lambda)}-\Pi_{S, 1}(0)-\Pi_{B, 1}(0)\left(1-C^{*}(\lambda)\right) \\
& \Pi_{B, 3}(0)=\frac{\Pi_{B, 2}(0)}{B^{*}(\lambda)}+\lambda \frac{\Pi_{B, 1}(0)}{B^{*}(\lambda)} \frac{B^{*(1)}(\lambda)}{B^{*}(\lambda)}-\Pi_{S, 2}(0) \\
& \Pi_{B, i}(0)=\frac{\Pi_{B, i-1}(0)}{B^{*}(\lambda)}-\lambda \frac{\Pi_{B, i-2}^{*}(\lambda)}{B^{*}(\lambda)}-\Pi_{S, i-1}(0), \quad \text { for } 4 \leq i \leq K .
\end{aligned}
$$

From (50), we notice that the r.h.s. of (53) contains the terms $\Pi_{B, j}(0)(1 \leq j \leq i-1)$. Together with the fact that we have already expressed $\Pi_{S, i}(0)(1 \leq i \leq K)$ in terms of $\Pi_{B, 1}(0)$, we can express $\Pi_{B, i}(0)(1 \leq i \leq K)$ in terms of $\Pi_{B, 1}(0)$ by using $(51),(52)$ and (53) $(4 \leq i \leq K)$ recursively. Next we substitute $s=0$ into (10) and (11). These equations respectively yield

$$
\begin{aligned}
& \lambda \Pi_{B, 1}^{*}(0)=-C^{*}(\lambda) \Pi_{B, 1}(0)+\Pi_{B, 2}(0)+\Pi_{S, 1}(0) \\
& \lambda \Pi_{B, i}^{*}(0)=-\Pi_{B, i}(0)+\lambda \Pi_{B, i-1}^{*}(0)+\Pi_{B, i+1}(0)+\Pi_{S, i}(0), \quad \text { for } 2 \leq i \leq K-1 .
\end{aligned}
$$

Since we have $\Pi_{B, i}(0)(1 \leq i \leq K)$ in terms of $\Pi_{B, 1}(0)$, we can easily express $\Pi_{B, i}^{*}(0)(0 \leq$ $i \leq K-1)$ in terms of $\Pi_{B, 1}(0)$ using (54) and (55). Differentiating (10), (11) and (12), and then substituting $s=0$ yield

$$
\begin{aligned}
\lambda \Pi_{B, 1}^{*(1)}(0) & =\Pi_{B, 1}^{*}(0)+\left(\Pi_{B, 2}(0)+\Pi_{S, 1}(0)+\Pi_{B, 1}(0)\left(1-C^{*}(\lambda)\right)\right) B^{*(1)}(0) \\
\lambda \Pi_{B, i}^{*(1)}(0) & =\Pi_{B, i}^{*}(0)+\lambda \Pi_{B, i-1}^{*(1)}(0)+\left(\Pi_{B, i+1}(0)+\Pi_{S, i}(0)\right) B^{*(1)}(0), \text { for } 2 \leq i \leq K-1 \\
-\Pi_{B, K}^{*}(0) & =\lambda \Pi_{B, K-1}^{*(1)}(0)+\Pi_{S, K}(0) B^{*(1)}(0) .
\end{aligned}
$$

From these results, we can express $\Pi_{B, K}^{*}(0)$ in terms of $\Pi_{B, 1}(0)$

$$
\Pi_{B, K}^{*}(0)=-\left\{\sum_{i=1}^{K-1} \Pi_{B, i}^{*}(0)+\left(\sum_{i=2}^{K} \Pi_{B, i}(0)+\sum_{i=1}^{K} \Pi_{S, i}(0)+\Pi_{B, 1}(0)\left(1-C^{*}(\lambda)\right)\right) B^{*(1)}(0)\right\}
$$


4.5 Obtaining $\Pi_{B, 1}(0)$

In the previous section we have $\Pi_{V, i}(0)(0 \leq i \leq K), \Pi_{V, i}^{*}(0)(0 \leq i \leq K), \Pi_{S, i}(0)(1 \leq i \leq$ $K), \Pi_{S, i}^{*}(0)(1 \leq i \leq K), \Pi_{B, i}(0)(1 \leq i \leq K)$ and $\Pi_{B, i}^{*}(0)(1 \leq i \leq K)$ in terms of $\Pi_{B, 1}(0)$. From the normalization condition

$$
\Pi_{0}^{*}+\Pi_{C, 0}^{*}(0)+\sum_{i=0}^{K} \Pi_{V, i}^{*}(0)+\sum_{i=1}^{K} \Pi_{S, i}^{*}(0)+\sum_{i=1}^{K} \Pi_{B, i}^{*}(0)=1
$$

we obtain $\Pi_{B, 1}(0)$. Hence, the system state probabilities immediately follow.

\section{Performance measures}

In this section, we focus on three important performance measures of the system from an application point of view. One is the loss probability of an arbitrary customer and the others are the set-up rate and the waiting time distribution of a customer which is accepted by the system.

\subsection{The loss probability}

We consider the probability $P_{\text {loss }}$ that an arbitrary customer is lost because of blocking and express it in two ways. The first approach is based on the Poisson Arrivals See Time Averages (PASTA) property. Since our arrival process is Poissonian, the probability that an arbitrary customer is blocked equals the probability that the number of customers in the system is $\mathrm{K}$ at an arbitrary time. Therefore, $P_{\text {loss }}$ is simply obtained as

$$
P_{\text {loss }}=\Pi_{S, K}^{*}(0)+\Pi_{V, K}^{*}(0)+\Pi_{B, K}^{*}(0) .
$$

On the other hand, if we apply Little's law to the server (excluding the waiting room), we have

$$
\lambda\left(1-P_{\text {loss }}\right)\left(-B^{*(1)}(0)\right)=\operatorname{Prob}[\text { The server is busy }]=\sum_{i=1}^{K} \Pi_{B, i}^{*}(0)
$$

from which we have

$$
P_{\text {loss }}=1-\frac{\sum_{i=1}^{K} \Pi_{B, i}^{*}(0)}{\lambda\left(-B^{*(1)}(0)\right)}
$$

\subsection{The set-up rate}

The set-up rate, $\gamma_{\text {set-up }}$ is defined as the number of set-up periods per unit time, which is of interest from the SVCC-operation point of view. This is obtained as

$$
\gamma_{s e t-u p}=\frac{\sum_{i=1}^{K} \Pi_{S, i}^{*}(0)}{-S^{*(1)}(0)}
$$

\subsection{Waiting time distribution}

We derive the distribution of the waiting time. First we observe an arriving customer classified by the system state in which it arrives. We call an idle period as the period during which the system is neither closing-down, setting-up, taking a vacation nor serving. In the idle period, the arriving customer is served after a set-up period. On the other hand, the arriving customer is served immediately during close-down period. In a vacation period, the arriving customer is served after the vacation period ends and the all customers in the queue at the arriving point are served followed by a set-up period. In the set-up period, the arriving customer is served after the set-up period ends and the all customers in the queue at the arriving point are served. In the busy period, the arriving customer is served after 
the service undertaking is complete and the all customers in the queue at the arriving point are served. From these observations, we obtain the LST of the waiting time of an arbitrary customer as follows.

$$
\begin{aligned}
W^{*}(s)=\frac{1}{1-P_{\text {loss }}}\{ & \Pi_{C, 0}^{*}(0)+\Pi_{0}^{*} S^{*}(s)+\sum_{i=0}^{K-1} \Pi_{V, i}^{*}(s)\left[S^{*}(s)\right]\left[B^{*}(s)\right]^{i} \\
& \left.+\sum_{i=1}^{K-1} \Pi_{S, i}^{*}(s)\left[B^{*}(s)\right]^{i}+\sum_{i=1}^{K-1} \Pi_{B, i}^{*}(s)\left[B^{*}(s)\right]^{i-1}\right\} .
\end{aligned}
$$

Differentiating (65) by $s$ and substituting $s=0$, we have the mean waiting time, $\mathbf{E}(W)$. This is, however, also obtained by Little's law

$$
\mathbf{E}(W)=\frac{\mathbf{E}(L)}{\lambda^{\prime}}
$$

where $\mathbf{E}(L)$ is the mean queue length obtained as

$$
\mathbf{E}(L)=\sum_{i=0}^{K} i \cdot \Pi_{V, i}^{*}(0)+\sum_{i=1}^{K} i \cdot \Pi_{S, i}^{*}(0)+\sum_{i=1}^{K}(i-1) \cdot \Pi_{B, i}^{*}(0)
$$

and $\lambda^{\prime}$ is the effective arriving rate given by

$$
\lambda^{\prime}=\frac{\sum_{i=1}^{K} \Pi_{B, i}^{*}(0)}{-B^{*(1)}(0)}
$$

\section{Numerical results}

To illustrate how our analysis is numerically tractable, we show some numerical results under the following scenario.

- $B(x)=1-e^{-x}$

- $V(x)=1-e^{-x}$

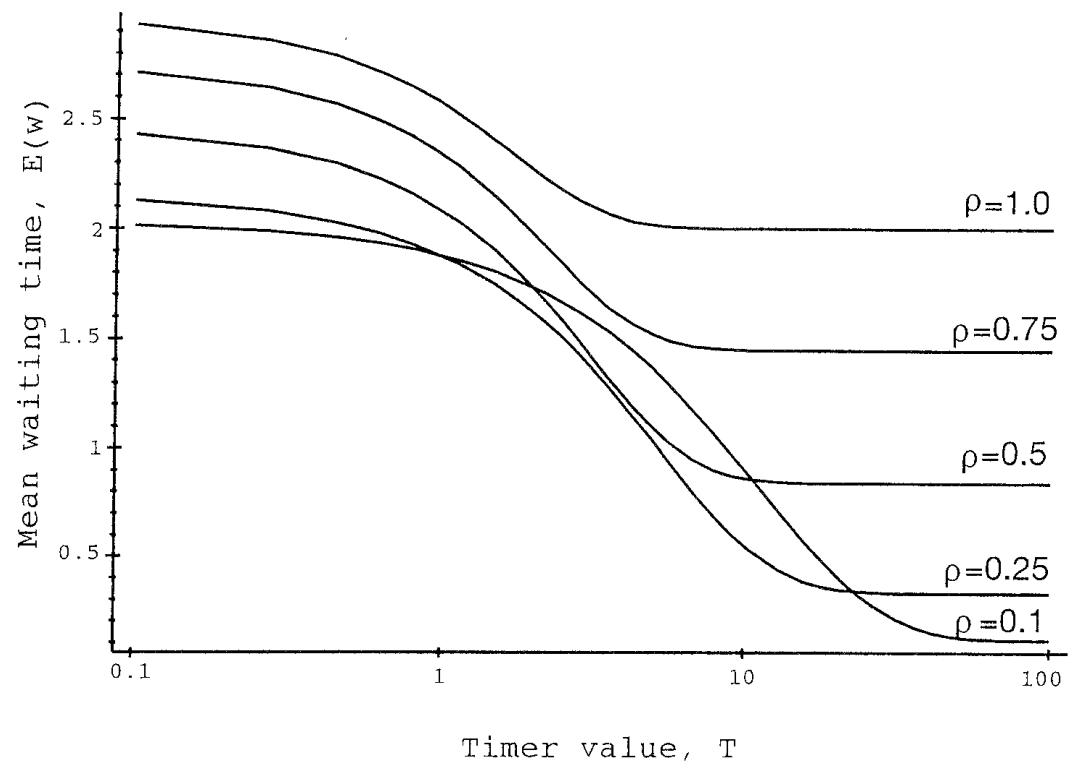

Figure 1: The mean waiting time 
- $S(x)=\int_{-\infty}^{x} \delta(x-2) d x$

- $C(x)=\int_{-\infty}^{x} \delta(x-T) d x$

- $K=5$

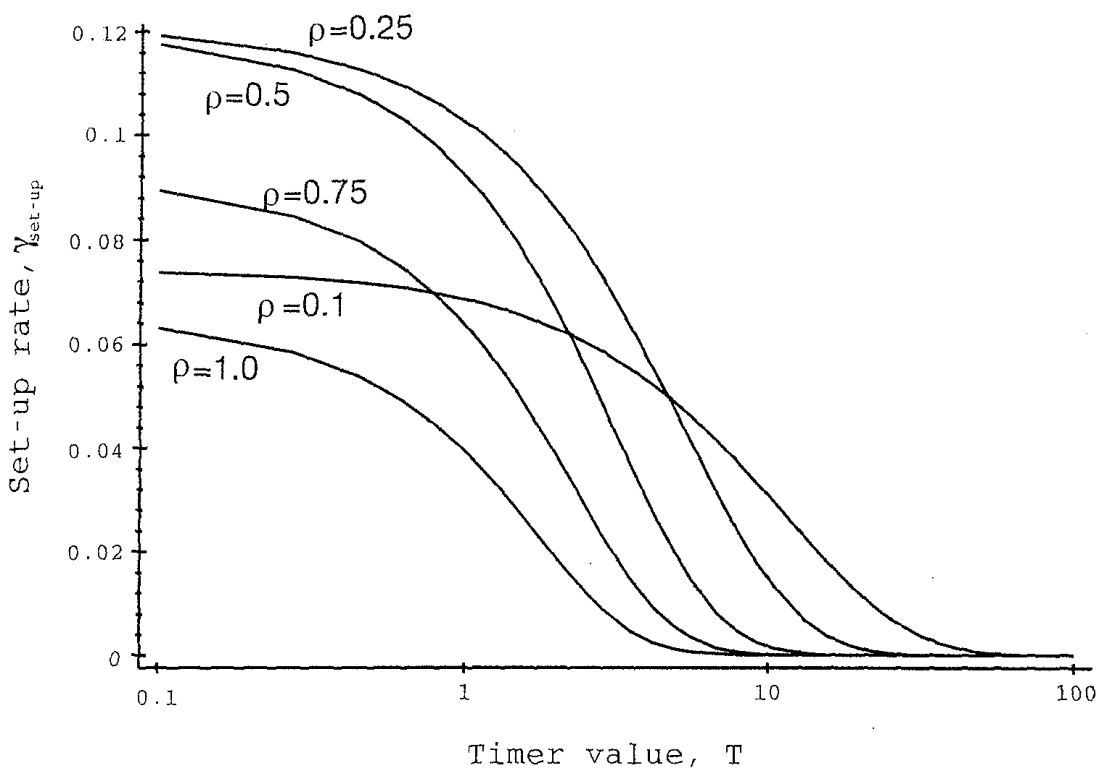

Figure 2: The set-up rate

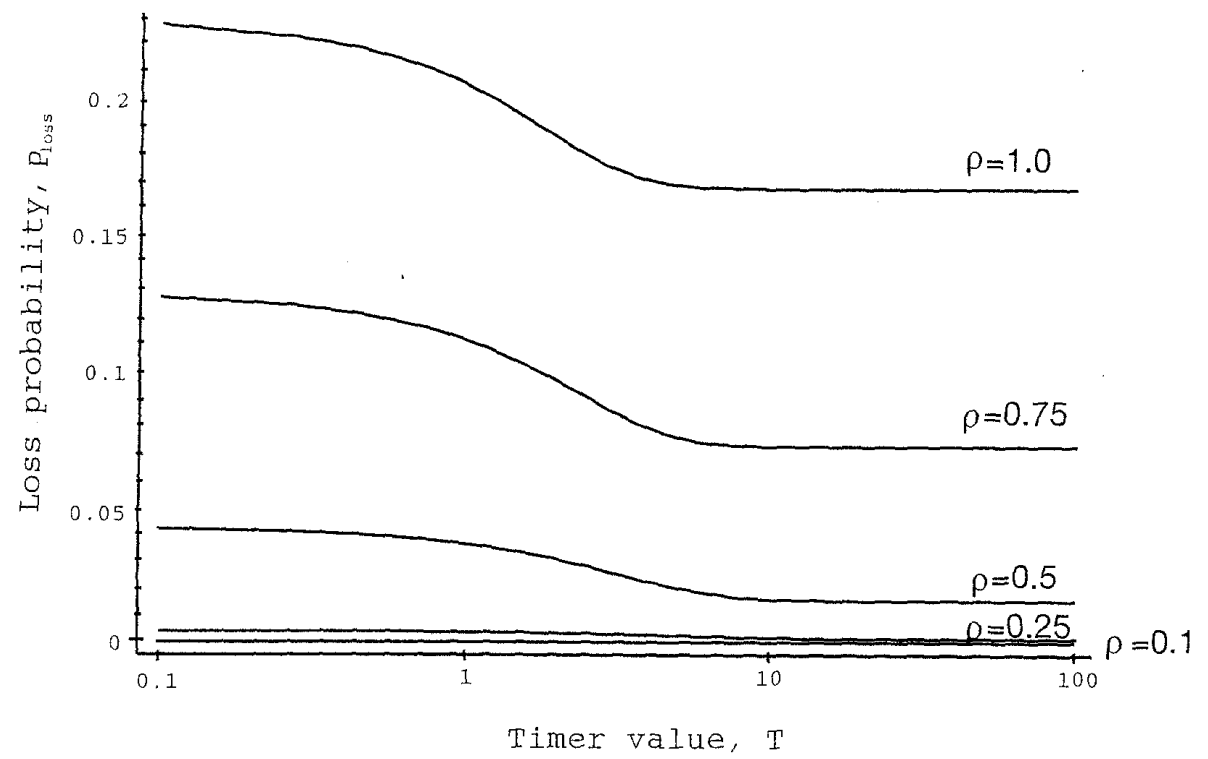

Figure 3: The loss probability

We have plotted the mean waiting times in Figure 1, the set-up rates in Figure 2 and loss probabilities in Figure 3 as a function of timer value $\mathrm{T}$ for different offered loads $\rho=$ $\lambda *\left(-B^{*(1)}(0)\right)$. In Figure 1, we observe that the mean waiting time gets longer for higher $\rho$ regardless of $\mathrm{T}$ in general but that it behaves in a different way for $\rho=0.1$. This comes from the fact in the following. The mean number of customers in the queue increases as $\rho$ becomes high. A longer mean waiting time is then required for higher $\rho$. For $\rho=0.1$, 
however the arrival rate is so small that the probability that the close-down time of $\mathrm{T}$ ends with arrivals is almost zero. Thus, almost all customers need a set-up time and the mean waiting time becomes larger.

Figure 2 shows that the set-up rate for higher $\rho$ is larger than that for lower $\rho$ except for $\rho=0.1$. Here is an explanation. The probability of an arrival during a close-down time is larger for higher $\rho$. Therefore, the set-up rate decreases for higher $\rho$. However for $\rho=0.1$, long idle periods decrease the set-up rate. From Figure 3 we see that the loss probability increases as $\rho$ increases and the length of close-down time has a small influence on the behavior of the loss probability.

\section{The infinite capacity model}

In this section, we show that our equation (65) for a finite capacity system is consistent with the corresponding result for the infinite capacity system as $\mathrm{K}$ tends to infinity. We define $W_{K \rightarrow \infty}^{*}(s)$ as the LST of the waiting time of an arbitrary customer for finite capacity system in which $\mathrm{K}$ tends to infinity and $W_{\infty}^{*}(s)$ as that for infinite capacity system.

\subsection{Waiting time analysis for infinite capacity model}

Let $\Pi(z)$ denote the distribution of the number of customers left at a service completion. Using the method of imbedded Markov chain (see Takagi [6]), we have

$$
\begin{aligned}
\Pi(z)= & \Pi_{0}\left(1-C^{*}(\lambda)\right) z B^{*}(\lambda-\lambda z) \frac{1}{z}+\Pi_{0} C^{*}(\lambda) V^{*}(\lambda) z S^{*}(\lambda-\lambda z) B^{*}(\lambda-\lambda z) \frac{1}{z} \\
& +\Pi_{0} C^{*}(\lambda)\left(1-V^{*}(\lambda)\right) \frac{V^{*}(\lambda-\lambda z)-V^{*}(\lambda)}{1-V^{*}(\lambda)} S^{*}(\lambda-\lambda z) B^{*}(\lambda-\lambda z) \frac{1}{z} \\
& +\frac{\Pi(z)-\Pi_{0}}{z} B^{*}(\lambda-\lambda z) .
\end{aligned}
$$

We denote by $\alpha(z)$ the generating function of the number of customers present at the beginning of a busy period. This $\alpha(z)$ is obtained as

$$
\alpha(z)=\left(1-C^{*}(\lambda)\right) z+C^{*}(\lambda)\left\{V^{*}(\lambda-\lambda z)-(1-z) V^{*}(\lambda)\right\} S^{*}(\lambda-\lambda z) .
$$

Using the equation above, we have

$$
\Pi(z)=\Pi_{0} \alpha(z) B^{*}(\lambda-\lambda z) \frac{1}{z}+\frac{\Pi(z)-\Pi_{0}}{z} B^{*}(\lambda-\lambda z) .
$$

Solving this equation, we obtain

$$
\Pi(z)=\frac{\Pi_{0}[1-\alpha(z)] B^{*}(\lambda-\lambda z)}{B^{*}(\lambda-\lambda z)-z} .
$$

Here, $\Pi_{0}$ is determined by the normalization condition $\Pi(1)=1$, so that

$$
\Pi_{0}=\frac{1-\lambda\left(-B^{*(1)}(0)\right)}{\alpha^{(1)}(1)} .
$$

Under the FIFO discipline, the customers who arrive at the system while a customer stays in the system are nothing but those left behind when he leaves the system. The generating functions of the number of customers arriving at the system during the waiting time and the service time are respectively given by $W_{\infty}^{*}(\lambda-\lambda z)$ and $B^{*}(\lambda-\lambda z)$. Therefore, we have

$$
\Pi(z)=W_{\infty}^{*}(\lambda-\lambda z) B^{*}(\lambda-\lambda z) .
$$


Substituting $s=\lambda-\lambda z$, and solving for $W_{\infty}^{*}(s)$, we get

$$
W_{\infty}^{*}(s)=\frac{\lambda[1-\alpha(1-s / \lambda)]}{s\left(\alpha^{(1)}(1)\right)} \frac{s\left(1-\lambda\left(-B^{*(1)}(0)\right)\right)}{s-\lambda+\lambda B^{*}(s)}=\frac{\lambda[1-\alpha(1-s / \lambda)]}{s\left(\alpha^{(1)}(1)\right)} W_{M / G / 1}^{*}(s)
$$

where $W_{M / G / 1}^{*}(s)$ is the LST of the waiting time of an arbitrary customer for the corresponding $\mathrm{M} / \mathrm{G} / 1$ system without any vacation, set-up and close-down times. Equation (72) then shows a stochastic decomposition result as in $[1,5,6]$.

\subsection{Limiting behavior of waiting time for our system as $K \rightarrow \infty$}

We introduce the generating functions

$$
\begin{aligned}
& \Pi_{V}(z, 0)=\sum_{i=0}^{\infty} \Pi_{V, i}(0) z^{i} \\
& \Pi_{V}(z, s)=\sum_{i=0}^{\infty} \Pi_{V, i}(s) z^{i} .
\end{aligned}
$$

We define $\Pi_{S}(z, 0), \Pi_{S}(z, s), \Pi_{B}(z, 0)$ and $\Pi_{B}(z, s)$ similarly. From (4), (5), (7), (8), (10) and (11), we have

$$
\begin{aligned}
(\lambda-\lambda z-s) \Pi_{V}^{*}(z, s)= & -\Pi_{V}(z, 0)+\Pi_{B, 1}(0) C^{*}(\lambda) V^{*}(s) \\
(\lambda-\lambda z-s) \Pi_{S}^{*}(z, s)= & -\Pi_{S}(z, 0)+\Pi_{V}(z, 0) S^{*}(s)+(z-1) \Pi_{V, 0}(0) S^{*}(s) \\
(\lambda-\lambda z-s) \Pi_{B}^{*}(z, s)= & -\Pi_{B}(z, 0)+\frac{\Pi_{B}(z, 0)}{z} B^{*}(s)+\Pi_{S}(z, 0) B^{*}(s) \\
& +\Pi_{B, 1}(0)\left(z-1-z C^{*}(\lambda)\right) B^{*}(s)
\end{aligned}
$$

Substituting $s=\lambda-\lambda z$ into the above equations, we have

$$
\begin{aligned}
\Pi_{V}(z, 0) & =\Pi_{B, 1}(0) C^{*}(\lambda) V^{*}(\lambda-\lambda z) \\
\Pi_{S}(z, 0) & =\Pi_{B, 1}(0) C^{*}(\lambda)\left\{V^{*}(\lambda-\lambda z)-(1-z) V^{*}(\lambda)\right\} S^{*}(\lambda-\lambda z) \\
\Pi_{B}(z, 0) & =\frac{z \Pi_{B, 1}(0)(\alpha(z)-1)}{z-B^{*}(\lambda-\lambda z)} B^{*}(\lambda-\lambda z) .
\end{aligned}
$$

Using these results, we get

$$
\begin{aligned}
& \Pi_{V}^{*}(z, s)=\frac{\Pi_{B, 1}(0) C^{*}(\lambda)\left\{V^{*}(s)-V^{*}(\lambda-\lambda z)\right\}}{\lambda-\lambda z-s} \\
& \Pi_{S}^{*}(z, s)=\frac{\Pi_{B, 1}(0) C^{*}(\lambda)\left\{V^{*}(\lambda-\lambda z)-(1-z) V^{*}(\lambda)\right\}\left\{S^{*}(s)-S^{*}(\lambda-\lambda z)\right\}}{\lambda-\lambda z-s} \\
& \Pi_{B}^{*}(z, s)=\frac{\Pi_{B, 1}(0)(1-\alpha(z))}{\lambda-\lambda z-s} \frac{z\left\{B^{*}(s)-B^{*}(\lambda-\lambda z)\right\}}{B^{*}(\lambda-\lambda z)-z} .
\end{aligned}
$$

We finally derive $W_{K \rightarrow \infty}^{*}(s)$ as follows

$$
\begin{aligned}
W_{K \rightarrow \infty}^{*}(s) & =\Pi_{C, 0}^{*}(0)+\Pi_{0}^{*} S^{*}(s)+\Pi_{V}^{*}\left(B^{*}(s), s\right)\left[S^{*}(s)\right]+\Pi_{S}^{*}\left(B^{*}(s), s\right)+\frac{\Pi_{B}^{*}\left(B^{*}(s), s\right)}{B^{*}(s)} \\
& =\frac{\Pi_{B, 1}(0)}{\lambda} \times \frac{-\lambda+\left(1-C^{*}(\lambda)\right)(\lambda-s)+C^{*}(\lambda)\left(\lambda V^{*}(s)-s V^{*}(\lambda)\right) S^{*}(s)}{\left(\lambda-\lambda B^{*}(s)-s\right)} \\
& =\frac{\Pi_{B, 1}(0)}{\lambda} \times \frac{\lambda(1-\alpha(1-s / \lambda))}{s-\lambda+\lambda B^{*}(s)}
\end{aligned}
$$


From the normalization condition,

$$
\Pi_{C, 0}^{*}(0)+\Pi_{0}^{*}+\Pi_{V}^{*}(1,0)+\Pi_{S}^{*}(1,0)+\Pi_{B}^{*}(1,0)=1,
$$

$\Pi_{B, 1}(0)$ is given by

$$
\Pi_{B, 1}(0)=\lambda \frac{1-\lambda\left(-B^{*(1)}(0)\right)}{\alpha^{(1)}(1)}
$$

from which we have shown

$$
W_{K \rightarrow \infty}^{*}(s)=W_{\infty}^{*}(s)
$$

\section{Remarks}

1. Notice that $\frac{1}{\lambda} \Pi_{B, 1}(0)=\Pi_{0}$. Here is an explanation. $\Pi_{0}$ is the probability that the number of customers left in the system is 0 when a customer leaves the system. Since we assume the queue size changes by unit jumps, this is consistent with the probability that an arbitrary customer finds the number of customers in the system is 0 . Recalling that the arriving process is Poissonian, this probability equals the probability that the system is empty at an arbitrary time (PASTA). On the other hand $\Pi_{B, 1}(0)$ stands for the instance that the system becomes empty and the mean length of the period that the system remains empty is given by $1 / \lambda$. The system state is either closing-down, taking a vacation, or idle. Therefore, $\frac{1}{\lambda} \Pi_{B, 1}(0)$ also gives the probability that the system is empty at an arbitrary time.

2. For non-vacation system $(V=0)$, our model reduces to that in Hassan and Atiquzzaman [3]. Our analysis developed here is exact, while the queueing analysis in [3] is an approximation by the $\mathrm{M} / \mathrm{G} / 1$ and heuristic results.

\section{References}

[1] B. Doshi, Single server queues with vacations, Stochastic Analysis of Computer Communication Systems, H. Takagi (editor), 217-265, Elsevier Science Publishers B.V., Amsterdam (1990).

[2] A. Frey and $\mathrm{Y}$. Takahashi, A note on an $\mathrm{M} / \mathrm{G} / 1 / \mathrm{N}$ queue with vacation time and exhaustive service discipline, Opns. Res. Letters, 21, 95-100 (1997).

[3] M. Hassan and M. Atiquzzaman, A delayed vacation model of an M/G/1 queue with applications to SVCC-based ATM networks, IEICE Trans. Commun., E80-B, 317-323 (1997).

[4] T.T. Lee, M/G/1/N queue with vacation time and exhaustive service discipline, Opns. Res., 32, 774-784 (1984).

[5] M. Miyazawa, Decomposition formulas for single server queues with vacations: A unified approach by the rate conservation law, Stochastic Models, 10, 389-413 (1994).

[6] H. Takagi, Queueing Analysis, A Foundation of Performance Evaluation, Vol.1: Vacation and Priority Systems, Elsevier Science Publishers B.V., Amsterdam (1991).

Yoshitaka Takahashi

NTT Multimedia Networks Laboratories

9-11, 3-Chome, Midori, Musashino, Tokyo 180, Japan

E-mail: yoshi@hashi.tnl.ntt.co.jp 